\title{
Criotolerancia del Semen Equino, Estabilidad Oxidativa y Componentes del Plasma Seminal
}

\author{
Cryotolerance of Stallion Semen, Oxidative Stability and Components \\ of Seminal Plasma
}

\author{
Alexandra Usuga S. ${ }^{1,4}$, Giovanni Restrepo B. ${ }^{2}$, Benjamín A. Rojano ${ }^{3}$
}

\section{Resumen}

\begin{abstract}
Se desconoce en gran medida el aporte del plasma seminal a la criotolerancia del semen equino. Algunos componentes del plasma seminal son conocidos por su acción antioxidante y, en consecuencia, podrían influir en la estabilidad oxidativa de los lípidos y las proteínas del semen. Esta investigación tuvo como objetivo evaluar la relación entre la estabilidad oxidativa y algunos componentes del plasma seminal con la criotolerancia del semen equino. En el plasma seminal de 30 caballos se evaluó el contenido de vitamina $\mathrm{C}(\mathrm{VC})$ por HPLC, proteínas totales (PT) por Bradford, y la oxidación proteica (OP) y lipídica (PL) por medición de carbonilos y el ensayo TBARS, respectivamente. El semen se congeló con $10 \%$ de plasma seminal y se evaluó la calidad seminal posdescongelación. Se realizó el ajuste de modelos lineales generalizados (GLM), análisis de regresión y correlación y la comparación de medias por Tukey. Se encontraron promedios de $7.13 \mathrm{mg}$ BSA $/ \mathrm{ml}$ y $6.05 \mathrm{mg} / \mathrm{l}$ para PT y VC, respectivamente. Los resultados de oxidación de lípidos y proteínas fueron de $3.27 \mathrm{nmol} \mathrm{MDA} / 1$ y $0.03 \mathrm{nmol}$ carbonilos $/ \mathrm{g}$ de proteína, respectivamente. Se encontraron asociaciones entre VC, PT, OP y PL con varios parámetros de calidad seminal posdescongelación $(\mathrm{p}<0.05)$. Se concluye que los niveles de vitamina $\mathrm{C}$, proteínas totales y oxidación del plasma seminal están asociados con la criotolerancia del semen equino.
\end{abstract}

Palabras clave: vitamina C, estrés oxidativo, congelación, espermatozoides

${ }^{1}$ Facultad de Medicina Veterinaria y Zootecnia, Universidad CES, Medellín, Colombia

${ }^{2}$ Facultad de Ciencias Agrarias, ${ }^{3}$ Facultad de Ciencias, Universidad Nacional de Colombia Sede Medellín, Colombia

${ }^{4}$ E-mail: ausuga@ces.edu.co

Recibido: 2 de febrero de 2016

Aceptado para publicación: 8 de mayo de 2016 


\begin{abstract}
Contribution of seminal plasma on stallion semen cryotolerance is largely unknown. Some components of seminal plasma are known for their antioxidant effect and therefore may influence the oxidative stability of lipids and proteins of semen. The aim of this research was to evaluate the relationship between the oxidative stability and some components of seminal plasma with stallion semen cryotolerance. In seminal plasma of 30 horses were assessed vitamin C content (VC) by HPLC, total protein (TP) by Bradford, and protein and lipid oxidation (PO and LP) by TBARS and carbonyls tests, respectively. The semen was frozen with $10 \%$ of seminal plasma and post-thaw semen quality was evaluated. Adjustment of generalized linear models (GLM), regression and correlation analysis, and comparison of means by Tukey were performed. Averages of $7.13 \mathrm{mg}$ $\mathrm{BSA} / \mathrm{ml}$ and $6.05 \mathrm{mg} / \mathrm{l}$ for PT and VC respectively were found. Results of lipids and proteins oxidation were $3.27 \mathrm{nmol} \mathrm{MDA} / 1$ and $0.03 \mathrm{nmol}$ carbonyls $/ \mathrm{g}$ protein, respectively. Association between VC, TP, LP and PO with various parameters of post-thawing semen quality were found $(\mathrm{p}<0.05)$. It is concluded that levels of total protein, vitamin $\mathrm{C}$ and seminal plasma oxidation are associated with stallion semen cryotolerance.
\end{abstract}

Key words: vitamin C, oxidative stress, freezing, spermatozoa

\section{INTRODUCCIÓN}

El estrés oxidativo se ha identificado como una de las principales causas de baja fertilidad seminal (Aitken, 2006; Moein et al., 2007; Ball 2008). Los espermatozoides equinos, por su alto contenido de ácidos grasos poliinsaturados en la membrana plasmática, son propensos al ataque de los radicales libres durante los procesos de congelación (García et al., 2011), donde se genera un incremento en los niveles de especies reactivas de oxígeno (ERO) y la peroxidación de los lípidos de la membrana (Giesecke et al., 2010; Vasconcelos et al., 2013). Altos niveles de ERO también pueden generar la oxidación de proteínas, conduciendo a la producción de grupos carbonilos, los cuales han sido descritos como biomarcadores para predecir el daño oxidativo proteico y se les ha asociado con la presencia de defectos severos en la función de los espermatozoides (Morte et al., 2008). Esto ha limitado el uso del semen equino criopreservado con fines de inseminación artificial debido a la baja fertilidad obtenida (Watson, 2000; Ortega et al., 2009; Heckenbichleret al., 2011).
Entre los componentes del plasma seminal equino, se han identificado algunos antioxidantes enzimáticos y no enzimáticos, los cuales tendrían la función de proteger los espermatozoides de los efectos nocivos de las ERO (Kankofer et al., 2005; Pesch et al., 2006; Waheed et al., 2013). No obstante, en los procesos de criopreservación se realiza usualmente la extracción del plasma seminal debido al efecto deletéreo que presenta sobre la capacitación de los espermatozoides (Kareskoski y Katila, 2008; Guasti et al., 2012).

En los últimos años se ha reportado que la suplementación con pequeñas cantidades de plasma seminal en los procesos de criopreservación podría reducir la peroxidación lipídica (Pizarro et al., 2013) y, por tanto, mejorar la calidad del semen posdescongelación (Katila et al., 2002; Moore et al., 2005). Así mismo, la adición de plasma seminal heterólogo (es decir, de equinos de alta congelabilidad) al semen de reproductores de baja congelabilidad tendría un efecto beneficioso en los parámetros de calidad seminal, lo cual sugiere que la composición del plasma seminal podría asociarse a la criotolerancia (Aurich et al., 1996). 
Por otro lado, la presencia de algunas moléculas como vitaminas y enzimas en el plasma seminal han sido reconocidas por su funcionalidad como antioxidantes (Bustamante, 2006; Vasconcelos et al., 2013; Waheed et al., 2013). La investigación en criopreservación de semen equino ha tenido importantes avances; sin embargo, existen vacíos de conocimiento en cuanto a su asociación con la reducción del estrés oxidativo durante la criopreservación, y respecto a su consecuente relación con la calidad seminal. El objetivo de esta investigación fue evaluar la asociación de algunos componentes del plasma seminal y su estabilidad oxidativa, con la calidad del semen equino criopreservado.

\section{Materiales y Métodos}

\section{Colecta y Evaluación de Semen Equino}

Se trabajó con 30 equinos (Equus caballus) de la raza Criollo Colombiano. Los equinos tenían entre 2 y 10 años de edad, condición corporal de 5 a 8 (escala 1-9) (Warren, 2009), y estaban sometidos a un régimen de una colecta semanal como mínimo.

Para efectos del estudio se utilizó una colecta de semen (fracción espermática) por animal mediante el método de vagina artificial, empleando una vagina modelo Missouri (Minitube). La fracción en gel del eyaculado fue removida por filtración. El semen recién recolectado fue diluido en proporción 1:1 en un diluyente a base de caseinatos, azúcares y antibióticos (EquiPlus ${ }^{\circledR}$, Minitube) a $37^{\circ} \mathrm{C}$, y se transportó a una temperatura de $5^{\circ} \mathrm{C}$ en un dispositivo de refrigeración (Equitainer ${ }^{\circledR}$, Hamilton Research Inc). Antes de la dilución del semen, una fracción del eyaculado fue centrifugada durante $15 \mathrm{~min}$ a $850 \mathrm{~g}$ con el fin de recuperar el plasma seminal, el cual se transportó igualmente a $5{ }^{\circ} \mathrm{C}$.

En cada eyaculado se determinó el volumen con una probeta graduada, la concentración mediante un fotómetro Spermacue ${ }^{\circledR}$
(Minitube) y la movilidad espermática utilizando un microscopio de contraste de fase Eclipse E200 (Nikon Inc.), obteniendo el promedio de cinco campos de observación (400X).

\section{Cuantificación de las Proteínas Totales}

La cuantificación de proteínas del plasma seminal se realizó mediante el método de Bradford, el cual se basa en el cambio de color de Coomassie Brilliant Blue G-250 en respuesta a diferentes concentraciones de proteínas. La unión del colorante con las proteínas se evaluó por espectrofotometría a $595 \mathrm{~nm}$, a temperatura ambiente durante 5 min. Se preparó una solución de BSA a una concentración de $1 \mathrm{mg} / \mathrm{ml}$ y se realizaron diluciones para la construcción de una curva patrón (Bradford, 1976).

\section{Medición de Vitamina C}

El contenido de vitamina $\mathrm{C}$ (ácido ascórbico, VC) se evaluó por el método de cromatografía líquida de alto rendimiento HPLC, según el protocolo modificado de Novakova et al. (2008). Se utilizó un cromatógrafo líquido (Shimadzu, LC-20AD), equipado con un autoinyector SIL-20A/HT, un módulo de comunicación CBM-20A y un detector de fila de diodos (PDA) a $245 \mathrm{~nm}$. La cuantificación se llevó a cabo en una columna C-8 de dimensiones $(5 \mu \mathrm{m}) 250 * 4.6$. Como fase móvil se utilizó ácido fórmico $0.1 \%$. La razón de flujo de la fase móvil fue de $0.8 \mathrm{ml} / \mathrm{min}, 35{ }^{\circ} \mathrm{C}$ y condiciones isocráticas. Para la identificación y cuantificación del compuesto se construyó previamente una curva de calibración con ácido ascórbico grado HPLC como patrón.

\section{Evaluación de la Peroxidación Lipídica}

La evaluación de la peroxidación lipídica (PL) se realizó por la metodología modificada de las sustancias reactivas al ácido tiobarbitúrico-TBARS- (Serafini-Cessi y Cessi, 1968, Morte et al., 2008); para lo cual $50 \mu 1$ de semen fue incubado por $1 \mathrm{~h}$ a $37^{\circ} \mathrm{C}$, 
y luego enfriado en agua con hielo durante $10 \mathrm{~min}$. Posteriormente, se adicionaron $40 \mu \mathrm{l}$ de ácido tricloroacético (TCA, SigmaAldrich) al 15\%, y $80 \mu$ lde ácido tiobarbitúrico (TBA, Sigma-Aldrich) al 0.67\%. La mezcla fue agitada e incubada por $20 \mathrm{~min}$ a $90^{\circ} \mathrm{C}$, y enfriada en agua con hielo durante $10 \mathrm{~min}$. Se adicionaron $300 \mu l$ de butanol (SigmaAldrich) en cada muestra y se agitaron por inversión durante $1 \mathrm{~min}$. Finalmente, se tomaron $300 \mu \mathrm{l}$ de la capa superior de la muestra para servirla en una placa multipozo. El complejo fluorescente formado fue leído en un espectrofluorímetro LS 55 (Perkin Elmer) a una $\lambda$ de excitación $500 \mathrm{~nm}$ y slit de excitación 5 , y $\lambda$ de emisión $550 \mathrm{~nm}$ y un slit de emisión 15. Los resultados fueron expresados como equivalentes de malonaldehido (nmol $\mathrm{MDA} / \mathrm{ml}$ de muestra).

\section{Evaluación de la Oxidación Proteica}

La acumulación de proteínas oxidadas en el plasma seminal fue evaluada por el contenido de grupos carbonilo, que se cuantificó espectrofotométricamente a través de la reacción con DNPH (2,4-dinitrofenilhidrazina) a $360 \mathrm{~nm}$ (Fagan et al., 1999). Las muestras fueron centrifugadas y el sedimento se solubilizó en $1 \mathrm{ml}$ de ácido tricloroacético (TCA) y se centrifugó nuevamente $(1500 \mathrm{~g}$, 10 min). Los sedimentos se incubaron con $1 \mathrm{ml}$ de $10 \mathrm{mM}$ de DNPH (recién preparado en $2 \mathrm{MH}$ de ácido clorhídrico-HCL, en la oscuridad) durante $1 \mathrm{~h}$ a temperatura ambiente, con agitación cada $10 \mathrm{~min}$. Luego se añadió $1 \mathrm{ml}$ de TCA al $20 \%$ y las muestras se centrifugaron a $20000 \mathrm{~g}$ durante $3 \mathrm{~min}$. El sobrenadante fue decantado y el sedimento se mezcló con $1 \mathrm{ml}$ de una solución 1:1 de etanol: etil-acetato. Posteriormente el sedimento se incubó con $1 \mathrm{ml}$ de guanidina $6 \mathrm{M}$ (preparada en PBS, pH 6.5) durante $15 \mathrm{~min}$ a $37^{\circ} \mathrm{C}$ y se centrifugó a $1500 \mathrm{~g}$ durante $10 \mathrm{~min}$. Se recogió el sobrenadante y la oxidación de proteínas se estimó por espectrofotometría a $360 \mathrm{~nm}$.

\section{Criopreservación del Semen Equino}

La criopreservación del semen se realizó mediante un protocolo modificado de congelación rápida (Madeiros et al., 2002: Bustamante et al., 2009). El semen diluido fue centrifugado por $15 \mathrm{~min}$ a $280 \mathrm{~g}$. El precipitado fue suspendido en diluyente EquiPlus ${ }^{\circledR}$ suplementado con $4 \%$ de yema de huevo, $5 \%$ de N,N-dimetilformamida (Sigma-Aldrich) y $10 \%$ de plasma seminal equino, en cantidad suficiente para una concentración final de 100 x $10^{6}$ espermatozoides por ml. Posteriormente, el semen fue mantenido en refrigeración a $5{ }^{\circ} \mathrm{C}$ por 15 min y almacenado en pajillas de $0.5 \mathrm{ml}$ mediante un equipo automático de empaque y sellado de pajillas por ultrasonido (MRS1 Dual V2, IMV Technologies). Las pajillas fueron sometidas a vapores de nitrógeno líquido por $15 \mathrm{~min}$ y finalmente fueron almacenadas en un termo de nitrógeno líquido (IMV Technologies). Después de un periodo de dos semanas como mínimo, las pajillas fueron descongeladas en baño María a $37^{\circ} \mathrm{C}$ por 1 min para la evaluación seminal posdescongelación.

\section{Evaluación Seminal Posdescongelación}

\section{Movilidad}

Se determinó la movilidad de los espermatozoides mediante un sistema computarizado de análisis de semen (CASA). Para esto se utilizó un procedimiento modificado del reportado por Hidalgo et al. (2005), empleando un microscopio de contraste de fase (Nikon E200) con una cámara digital (Basler Scout SCA780) adaptada a un computador dotado del software Sperm Class Analyzer $\left(\mathrm{SCA}^{\circledR}\right)$ Motility and Concentration (Microptic SL). Se analizaron los parámetros de movilidad total (MT), movilidad progresiva (MP), velocidad rectilínea (VSL), velocidad curvilínea (VCL), velocidad media (VAP), amplitud del desplazamiento lateral de la cabeza (ALH) y frecuencia de batida (BCF). 


\section{Morfología}

La morfología espermática se evaluó mediante el conteo de 200 espermatozoides teñidos por la técnica de eosina-nigrosina modificada por Barth y Oko (1989) y Brito et al. (2011). Se observó la morfología individual de los espermatozoides al microscopio (1000X), clasificándolos como normales o anormales (Graham y Mocé, 2005).

\section{Vitalidad}

La vitalidad espermática se evaluó mediante el procedimiento descrito por Gamboa et al. (2010) con el kit Live/Dead (Molecular Probes Inc). Se incubó una suspensión de células $\left(20 \times 10^{6}\right.$ espermatozoides $\left./ \mathrm{ml}\right)$ en solución Hanks Heppes $(\mathrm{HH})$ con $1 \%$ de albúmina sérica bovina (BSA) por 5 min a $35^{\circ} \mathrm{C}$ con el fluorocromo para ácidos nucleicos SYBR14 (fluorescencia verde en todos los espermatozoides), a una concentración final de $6 \mathrm{mM}$. Luego fueron incubados de igual forma con el fluorocromo de ácidos nucleicos (fluorescencia roja en células muertas), yoduro de propidio (IP) a $0.48 \mathrm{mM}$.

Muestras de $5 \mu \mathrm{l}$ de espermatozoides fueron puestas en un portaobjetos de vidrio, con un respectivo cubreobjetos, y se contaron 200 células por microscopía de fluorescencia (Microscopio E200 con fluorescencia HBO, Nikon Inc) a longitudes de onda entre 550 y $595 \mathrm{~nm}$.

\section{Integridad de la membrana plasmática (HOS)}

Fue evaluada por la metodología de la prueba hipoosmótica (HOS), según Neild et al. (1999). Se tomaron $100 \mu 1$ de semen y se adicionaron a un tubo con $500 \mu 1$ de una solución hipoosmótica de sacarosa 5.4\% (100 $\mathrm{mOsmol} / \mathrm{l})$. La mezcla fue incubada a $38.5^{\circ} \mathrm{C}$ por 30 min y se evaluó el hinchamiento espermático de 100 células en un mínimo de cinco campos (400X), utilizando un microscopio de contraste de fase Eclipse E200
(Nikon Inc). Se determinó el porcentaje de células reaccionadas a partir de 200 espermatozoides contados.

\section{Análisis Estadístico}

Se ajustó un modelo lineal generalizado (GLM) para cada parámetro de calidad seminal (variable dependiente). Se calculó la distribución en cuartiles de los resultados para PT, VC, PL y OP, a partir de lo cual se establecieron tres niveles para cada variable (bajo, medio y alto). Las medias entre dichos niveles fueron comparadas mediante la prueba de Tukey. El modelo estadístico empleado fue:

$\mathrm{Y}_{\mathrm{ijklmno}}=\mu+\mathrm{E}_{\mathrm{i}}+\mathrm{PT}_{\mathrm{j}}+\mathrm{VC}_{\mathrm{k}}+\mathrm{PL}_{1}+\mathrm{OP}_{\mathrm{m}}+\mathrm{C}_{\mathrm{n}}+\mathrm{e}_{\mathrm{ijklmno}}$, donde:

$\mathrm{Y}_{\mathrm{ijklmn}}: \mathrm{MT}, \mathrm{MP}, \mathrm{VE}, \mathrm{MA}, \mathrm{HOS}, \mathrm{VCL}, \mathrm{VSL}, \mathrm{VAP}, \mathrm{ALH}$ o BCF

$\mu$ : Media común

$\mathrm{E}_{\mathrm{i}}$ : Efecto fijo del equino (i), $(\mathrm{i}=0 \ldots 30)$

$\mathrm{PT}_{\mathrm{j}}$ : Efecto fijo del nivel de proteínas totales $(\mathrm{j}),(\mathrm{j}=1-4)$

$\mathrm{VC}_{\mathrm{k}}$ : Efecto fijo del nivel de vitamina $\mathrm{C}(\mathrm{k}),(\mathrm{k}=1-4)$

$\mathrm{PL}_{1}$ : Efecto fijo del nivel de peroxidación lipídica (1), $(1=1-4)$

$\mathrm{OP}_{\mathrm{m}}$ : Efecto fijo del nivel de oxidación proteica $(\mathrm{m}),(\mathrm{m}=$ 1-4)

$\mathrm{C}_{\mathrm{n}}$ : Efecto fijo de la(s) covariable(s) de calidad seminal (n) $\mathrm{e}_{\mathrm{ijklmno}}$ : Error experimental

El análisis de correlación de Pearson se utilizó como criterio de elección de las covariables incluidas en cada modelo. La magnitud de la relación entre las variables fue evaluada mediante un análisis de regresión. El nivel de significancia para todos los análisis fue $\mathrm{p}<0.05$.

\section{Resultados y Discusión}

Los resultados para los componentes y estabilidad oxidativa del plasma seminal equino se presentan en el Cuadro 1. Los niveles de vitamina $\mathrm{C}$ fueron superiores a los reportados para caballos árabes, donde Waheed et al. (2013) reporta valores diferentes según la estación del año, variando de $0.84 \mathrm{mg} /$ 
Cuadro 1. Componentes y estabilidad oxidativa del plasma seminal equino $(n=30)$

\begin{tabular}{lccccc}
\hline Variable & Media & DE & CV & Mínimo & Máximo \\
\hline $\begin{array}{l}\text { Proteínas totales } \\
\quad(\mathrm{mg} \mathrm{BSA} / \mathrm{ml})\end{array}$ & 7.13 & 4.04 & 53.06 & 1.72 & 18.50 \\
$\begin{array}{l}\text { Vitamina C } \\
\quad(\mathrm{mg} / \mathrm{l})\end{array}$ & 6.05 & 1.47 & 24.55 & 3.79 & 10.04 \\
$\begin{array}{c}\text { Peroxidación lipídica } \\
\quad(\mathrm{nmmol} \text { MDA/l) }\end{array}$ & 3.27 & 1.90 & 54.89 & 0.97 & 10.99 \\
$\begin{array}{l}\text { Oxidación proteica } \\
\quad(\mathrm{nmol} \text { carbonilos / mg de } \\
\text { proteína) }\end{array}$ & 0.03 & 0.02 & 80.89 & 0.01 & 0.13 \\
\hline
\end{tabular}

DE: desviación estándar; CV: coeficiente de variación

Cuadro 2. Resultados de los modelos lineales generalizados (GLM) para los parámetros de calidad de semen equino $(n=30)$

\begin{tabular}{lcccl}
\hline Variable & Media & CV $(\%)$ & $\mathrm{R}^{2}$ & $\begin{array}{l}\text { Efectos } \\
(\mathrm{p}<0.05)\end{array}$ \\
\hline Movilidad total $(\%)$ & 48.40 & 8.63 & 0.96 & PT, OP \\
Movilidad progre siva (\%) & 18.87 & 9.62 & 0.97 & PT, OP, VC \\
VSL $(\mu \mathrm{m} / \mathrm{s})$ & 27.90 & 3.99 & 0.98 & $\begin{array}{l}\text { PT, OP, } \\
\text { VC, PL }\end{array}$ \\
VCL $(\mu \mathrm{m} / \mathrm{s})$ & 63.34 & 2.70 & 0.98 & PT, OP \\
VAP $(\mu \mathrm{m} / \mathrm{s})$ & 41.44 & 2.23 & 0.99 & PT, OP, \\
ALH $(\mu \mathrm{m})$ & 3.15 & 5.28 & 0.90 & OP, VC \\
BCF $(\mathrm{Hz})$ & 8.92 & 7.61 & 0.87 & VC \\
Vitalidad espermática $(\%)$ & 42.85 & 8.31 & 0.94 & OP \\
Morfología anormal $(\%)$ & 23.57 & 13.21 & 0.88 & PL \\
HOS $(\%)$ & 33.16 & 12.82 & 0.89 & \\
\hline
\end{tabular}

CV: Coeficiente de variación; $R^{2}$ : Coeficiente de determinación; VC: Vitamina C; PT: Proteínas totales; PL: Peroxidación lipídica; OP: Oxidación proteica; VSL: Velocidad rectilínea; VCL: Velocidad curvilínea; VAP: Velocidad media; ALH: Amplitud del desplazamiento lateral de la cabeza; BCF: Frecuencia de batida; HOS: Integridad de membrana plasmática

$1(2.12 \mathrm{nmol} / \mathrm{ml})$ en verano a $2.05 \mathrm{mg} / 1(5.13$ $\mathrm{nmol} / \mathrm{ml}$ ) en primavera; asimismo, reporta un contenido mayor de vitamina $\mathrm{C}$ en equinos mayores de 18 años de edad $(4.14 \pm 0.89$ $\mathrm{nmol} / \mathrm{ml})$ que en caballos jóvenes $(2.77 \pm 0.40$ $\mathrm{nmol} / \mathrm{ml})$.
En relación con la oxidación proteica del plasma seminal, Morte et al. (2008) encontraron en equinos fértiles y subfértiles valores de 2.52 y $3.40 \mathrm{nmol}$ carbonilos/mg de proteína, respectivamente, para la temporada reproductiva, y de 2.39 y $6.61 \mathrm{nmol}$ 
Cuadro 3. Comparación de medias (prueba de Tukey) de calidad seminal por niveles de estabilidad oxidativa y componentes del plasma seminal equino

\begin{tabular}{|c|c|c|c|c|c|c|c|c|c|c|c|}
\hline & vel & MT & MP & ISL & $\mathrm{VCL}$ & $\mathrm{P}$ & ALH & $\mathrm{BCF}$ & $\mathrm{E}$ & MA & IOS \\
\hline \multirow[t]{3}{*}{$\mathrm{PT}$} & Alto & $48.7^{b}$ & $19.3^{b}$ & $26.5^{b}$ & $60.6^{b}$ & $39.1^{\mathrm{a}}$ & $3.2^{\mathrm{a}}$ & $9.6^{\mathrm{a}}$ & $42.9^{b}$ & $21.1^{\mathrm{b}}$ & $32.0^{b}$ \\
\hline & Medio & $55.9^{\mathrm{a}}$ & $21.5^{\mathrm{a}}$ & $26.7^{\mathrm{ab}}$ & $66.4^{\mathrm{a}}$ & $42.1^{\mathrm{a}}$ & $3.3^{\mathrm{a}}$ & $9.0^{\mathrm{b}}$ & $48.7^{\mathrm{a}}$ & $22.2^{\mathrm{b}}$ & $36.9^{\mathrm{a}}$ \\
\hline & Bajo & $41.9^{c}$ & $16.2^{\mathrm{c}}$ & $29.7^{\mathrm{a}}$ & $62.5^{\mathrm{ab}}$ & $42.3^{\mathrm{a}}$ & $3.0^{\mathrm{b}}$ & $8.2^{\mathrm{c}}$ & $37.8^{\mathrm{c}}$ & $26.5^{\mathrm{a}}$ & $30.8^{\mathrm{b}}$ \\
\hline \multirow[t]{3}{*}{ PL } & Alto & $49.5^{\mathrm{a}}$ & $17.9^{\mathrm{b}}$ & $26.3^{\mathrm{b}}$ & $60.1^{\mathrm{b}}$ & $38.2^{\mathrm{b}}$ & $3.2^{\mathrm{a}}$ & $9.2^{\mathrm{a}}$ & $44.7^{\mathrm{b}}$ & $22.6^{\mathrm{b}}$ & $35.9^{\mathrm{a}}$ \\
\hline & Medic & $52.9^{\mathrm{a}}$ & $21.6^{\mathrm{a}}$ & $26.8^{\mathrm{ab}}$ & $65.6^{\mathrm{a}}$ & $41.4^{\mathrm{ab}}$ & $3.2^{\mathrm{a}}$ & $8.7^{\mathrm{b}}$ & $47.0^{\mathrm{a}}$ & $25.2^{\mathrm{a}}$ & $35.7^{\mathrm{a}}$ \\
\hline & Bajo & $44.6^{\mathrm{b}}$ & $17.7^{\mathrm{b}}$ & $29.8^{\mathrm{a}}$ & $64.2^{\mathrm{ab}}$ & $43.9^{\mathrm{a}}$ & $3.1^{\mathrm{a}}$ & $8.7^{\mathrm{b}}$ & $38.5^{\mathrm{c}}$ & $23.0^{\mathrm{b}}$ & $29.0^{\mathrm{b}}$ \\
\hline \multirow[t]{3}{*}{ OP } & Alto & $49.6^{\mathrm{a}}$ & $20.1^{\mathrm{b}}$ & $28.6^{\mathrm{a}}$ & $65.2^{\mathrm{a}}$ & $42.8^{\mathrm{a}}$ & $3.1^{\mathrm{b}}$ & $8.8^{\mathrm{a}}$ & $48.3^{\mathrm{a}}$ & $23.6^{\mathrm{ab}}$ & $35.4^{\mathrm{a}}$ \\
\hline & Medic & $52.8^{\mathrm{a}}$ & $21.4^{\mathrm{a}}$ & $26.2^{\mathrm{a}}$ & $65.5^{\mathrm{a}}$ & $41.0^{\mathrm{a}}$ & $3.3^{\mathrm{a}}$ & $9.0^{\mathrm{a}}$ & $42.2^{\mathrm{b}}$ & $25.4^{\mathrm{a}}$ & $35.7^{\mathrm{a}}$ \\
\hline & Bajo & $44.8^{\mathrm{b}}$ & $16.0^{\mathrm{c}}$ & $28.2^{\mathrm{a}}$ & $60.0^{\mathrm{b}}$ & $40.1^{\mathrm{a}}$ & $3.0^{c}$ & $8.8^{\mathrm{a}}$ & $39.9^{c}$ & $22.1^{\mathrm{b}}$ & $29.5^{b}$ \\
\hline \multirow[t]{3}{*}{$\mathrm{VC}$} & Alto & $44.0^{\mathrm{b}}$ & $18.1^{\mathrm{b}}$ & $29.6^{\mathrm{a}}$ & $61.1^{\mathrm{b}}$ & $41.5^{\mathrm{ab}}$ & $2.9^{\mathrm{b}}$ & $9.1^{\mathrm{a}}$ & $41.1^{b}$ & $24.4^{\mathrm{a}}$ & $30.8^{b}$ \\
\hline & Medio & $45.9^{\mathrm{b}}$ & $18.0^{\mathrm{b}}$ & $30.7^{\mathrm{a}}$ & $66.3^{\mathrm{a}}$ & $44.4^{\mathrm{a}}$ & $3.3^{\mathrm{a}}$ & $8.5^{\mathrm{b}}$ & $40.0^{\mathrm{b}}$ & $22.5^{\mathrm{b}}$ & $29.1^{\mathrm{b}}$ \\
\hline & Bajo & $54.3^{\mathrm{a}}$ & $20.1^{\mathrm{a}}$ & $24.2^{\mathrm{b}}$ & $62.7^{\mathrm{ab}}$ & $38.8^{\mathrm{b}}$ & $3.2^{\mathrm{a}}$ & $8.9^{\mathrm{ab}}$ & $46.5^{\mathrm{a}}$ & $23.5^{\mathrm{ab}}$ & $38.1^{\mathrm{a}}$ \\
\hline
\end{tabular}

${ }_{a, b, c}$ Superíndices diferentes dentro de columnas y grupos indic an diferencia significativa $(p<0.05)$

PT: Proteínas totales; PL: Peroxidación lipídica; OP: Oxidación proteica; VC: Vitamina C

MT: Movilidad total; MP: Movilidad progresiva; VSL: Velocidad rectilínea; VCL: Velocidad curvilínea; VAP: Velocidad media; ALH: Amplitud del desplazamiento lateral de la cabeza; BCF: Frecuencia de batida; VE: Vitalidad espermática; MA: Morfología anormal; HOS: Integridad de membrana plasmática

carbonilos/mg de proteína para la temporada no reproductiva. En todos los casos, esos valores superan los hallados en este estudio, donde el promedio para la OP fue de 0.03 nmol carbonilos/mg de proteína. Así mismo, estos autores reportaron valores para la peroxidación lipídica en caballos fértiles y subfértiles de 0.19 y $0.22 \mathrm{nmol} \mathrm{MDA} / \mathrm{mg}$ de proteína para la temporada reproductiva y de 0.15 y $0.22 \mathrm{nmol} \mathrm{MDA} / \mathrm{mg}$ de proteína para la temporada no reproductiva, lo cual sugiere que los procesos oxidativos del plasma seminal son superiores en los equinos de menor fertilidad.

La criopreservación del semen equino es conocida por la reducción de diferentes indicadores de calidad seminal, tales como la movilidad total, la movilidad progresiva, la permeabilidad de la membrana plasmática, la morfología e incluso la actividad mitocondrial (Parks y Graham, 1992; Brum et al., 2008). El Cuadro 2 muestra los resultados de los modelos ajustados para los parámetros de calidad del semen equino y el análisis de su asociación con los componentes y la estabilidad oxidativa del plasma seminal.

Algunos investigadores indican que el plasma seminal equino debe ser retirado antes de la criopreservación para evitar una disminución de la movilidad de los espermatozoides posdescongelación (Rigby et al., 2001; Kareskoski y Katila, 2008). Sin embargo, se ha observado efectos beneficiosos en la calidad seminal cuando se suplementa en cantidades menores de $20 \%$ (Alghamdi et al., 2002; Katila et al., 2002, 
Cuadro 4. Coeficientes de regresión (línea superior) y correlación (línea inferior) entre la estabilidad oxidativa y componentes del plasma seminal con parámetros de calidad seminal equino

\begin{tabular}{lllllllllll}
\hline & MT & MP & VSL & VCL & VAP & ALH & BCF & VE & MA & HOS \\
\hline PT & 0.24 & -0.01 & -0.28 & -0.43 & -0.34 & 0.01 & $0.09^{*}$ & 0.05 & $-0.46^{*}$ & -0.21 \\
& 0.05 & -0.01 & -0.13 & -0.13 & -0.12 & 0.08 & $0.23^{*}$ & 0.01 & $-0.23^{*}$ & -0.07 \\
PL & 1.35 & 0.30 & $-0.78^{*}$ & -0.91 & $-1.31^{*}$ & $0.04^{*}$ & $0.24^{*}$ & $1.32^{*}$ & 0.60 & $1.59^{*}$ \\
& 0.14 & 0.06 & $-0.16^{*}$ & -0.13 & $-0.22^{*}$ & $0.18^{*}$ & $0.29^{*}$ & $0.19^{*}$ & 0.14 & $0.27^{*}$ \\
OP & -64.0 & -25.7 & -25.1 & -9.75 & -25.5 & 0.10 & -3.10 & -65.7 & $66.8^{*}$ & 26.8 \\
& -0.09 & -0.07 & -0.07 & -0.01 & -0.06 & 0.01 & -0.05 & -0.13 & $0.22^{*}$ & 0.06 \\
VC & $-3.38^{*}$ & $-1.61^{*}$ & 0.53 & $-2.18^{*}$ & -0.67 & $-0.07^{*}$ & 0.03 & $-1.65^{*}$ & 0.04 & $-2.20^{*}$ \\
& $-0.28^{*}$ & $-0.24^{*}$ & 0.08 & $-0.24^{*}$ & -0.09 & $-0.26^{*}$ & 0.03 & $-0.19^{*}$ & 0.01 & $-0.29^{*}$ \\
\hline
\end{tabular}

* Coeficiente con $\mathrm{p}<0.05$

PT: Proteínas totales; PL: Peroxidación lipídica; OP: Oxidación proteica; VC: Vitamina C

MT: Movilidad total; MP: Movilidad progresiva; VSL: Velocidad rectilínea; VCL: Velocidad curvilínea; VAP: Velocidad media; ALH: Amplitud del desplazamiento lateral de la cabeza; BCF: Frecuencia de batida; VE: Vitalidad espermática; MA: Morfología anormal; HOS: Integridad de membrana plasmática

Moore et al., 2005), así como en la reducción de la peroxidación lipídica del semen equino criopreservado (Pizarro et al., 2013). En este estudio, los resultados encontrados para MT fueron superiores a los reportados por Moore et al. (2005) cuando se suplementó con plasma seminal al $10 \%$, mientras que fueron equiparables para la movilidad progresiva (Cuadro 2). Por el contrario, Morrell et al. (2014) reportaron resultados para MT de $79 \pm 1.6 \%$, MP de $53 \pm 1.5$, VCL de $123 \pm$ $1.7 \mu \mathrm{m} / \mathrm{s}$, VSL de $52 \pm 0.7 \mu \mathrm{m} / \mathrm{s}$ y VAP de 67 $\pm 0.8 \mu \mathrm{m} / \mathrm{s}$ cuando se suplementó con $5 \%$ de plasma seminal, los cuales son superiores a los encontrados en el presente estudio. Los modelos ajustados para la evaluación del semen equino criopreservado presentaron altos coeficientes de determinación $\left(\mathrm{R}^{2}\right)$, con lo cual se infiere que los efectos fijos y covariables considerados explican en una alta proporción la variabilidad para el parámetro estudiado (variable dependiente).
Se encontró un efecto significativo de las proteínas totales (PT) sobre MT, MP, VSL, VCL y VAP (Cuadro 2; $<<0.05$ ), resultados que difieren de los reportados por Guasti et al. (2014), quienes a pesar de encontrar un contenido por eyaculado de proteínas totales superior en caballos fértiles que en los subfértiles $(740.8 \pm 489.6$ vs $416.4 \pm 254.7$ $\mathrm{mg}$, respectivamente), no hallaron asociación sobre los parámetros de calidad seminal evaluados.

En el presente estudio, un nivel medio de proteínas arrojaron los mejores resultados para los parámetros de MT, MP, VE y HOS (Cuadro 3), probablemente debido a que un alto nivel de PT podría conducir a una mayor oxidación proteica, y a la consecuente reducción de la calidad seminal. Estudios similares donde se evaluó el efecto de la suplementación de proteínas del plasma seminal para mejorar la calidad del semen equino criopreservado 
presentan resultados poco consistentes. Así, Fagundes (2003) observó que la adición de proteínas de plasma seminal autólogo con masa molecular superior a $10 \mathrm{kDa}$ mejoró las características espermáticas posdescongelación, a diferencia de las proteínas con masa inferior a $10 \mathrm{kDa}$ que afectaron negativamente la viabilidad espermática, mientras que Pessanha et al. (2008) encontraron que los parámetros de calidad seminal fueron inferiores con la suplementación de proteínas del plasma seminal al 10 y $20 \%$ (con masa superior a $10 \mathrm{kDa}$ ) que aquellos en el grupo control.

Se evidenció una relación directa entre PT y BCF, así como una relación inversa con la morfología anormal (Cuadro 4). Este resultado podría ser explicado por una mayor presencia de proteínas con funciones antioxidantes, tales como las enzimas SOD, GPx, catalasa y transferrina, que por su acción sobre los radicales libres y sobre moléculas intermediarias de rutas de oxidación, como el hierro, podrían reducir los fenómenos oxidativos sobre el contenido total de proteínas (Kirschvink et al., 2008).

Se encontraron efectos significativos para la vitamina $\mathrm{C}$ sobre MP, VSL, VAP, ALH Y BCF (Cuadro 2; $p<0.05$ ) y coeficientes de correlación y regresión negativos para $\mathrm{MT}$, MP, VCL,ALH, VE yHOS (Cuadro 4; $<<0.05$ ). Lo anterior es apoyado por los hallazgos presentados en el Cuadro 3, ya que se encontró que un nivel bajo de vitamina $C$ en el plasma seminal equino arrojó resultados superiores $(\mathrm{p}<0.05)$ para los parámetros MT, MP, VE y HOS, en comparación con el nivel medio y alto para esta vitamina. Estos resultados coinciden con Waheed et al. (2013), quienes obtuvieron concentraciones menores de vitamina $\mathrm{C}$ en caballos de alta fertilidad, comparados con los de baja fertilidad.

Adicionalmente, se ha observado que la suplementación con altas concentraciones de ácido ascórbico para el proceso de criopreservación de semen equino presenta efectos negativos sobre la peroxidación lipídica de la membrana espermática $(\mathrm{p}<0.05)$ (Vasconcelos et al., 2013). Este efecto puede deberse a que la vitamina $C$ puede actuar como un pro-oxidante. En presencia de metales de transición, la vitamina $\mathrm{C}$ genera que algunos radicales se tornen más reactivos y destructivos, produciendo por consiguiente, más radicales libres. Adicionalmente, tiene la habilidad de promover la liberación de dichos metales de transición desde las proteínas, exacerbando este efecto (Michael et al., 2008; Vasconcelos et al., 2013). En humanos, el nivel de vitamina $C$ en espermatozoides de pacientes astenoteratozoospérmicos es dos veces mayor que en pacientes normozoospérmicos (Nouri et al., 2008). En el canino, Michael et al. (2008) reportaron que la suplementación con vitamina $\mathrm{C}$ no mostró una influencia positiva sobre la calidad del semen preservado a $4{ }^{\circ} \mathrm{C}$, ni sobre la inhibición en la producción de ERO, pero afectó la movilidad espermática.

Se encontró asociación de la peroxidación lipídica con los efectos VSL, VAP y MA (Cuadro 2), y coeficientes de correlación y regresión negativos para los dos primeros, indicando una relación inversa (Cuadro 4). Esto pudo deberse a que las ERO producidas durante el proceso de criopreservación pueden disminuir la movilidad seminal (Baumber et al., 2000), debido a la caída en los niveles de ATP (Vasconcelos et al., 2013), así como daños y pérdida de fluidez en la membrana de los espermatozoides debido a la peroxidación lipídica (Ballet al., 2000; Vasconcelos et al., 2013). Lo anterior podría explicar resultados superiores encontrados para VAP cuando se reportó un nivel bajo de PL, en comparación al nivel alto (Cuadro 3; $\mathrm{p}<0.05$ ). Adicionalmente, se ha observado una correlación positiva entre los niveles de peroxidación lipídica y el aumento de defectos morfológicos en espermatozoides bovinos (Nichi et al., 2006) $\mathrm{y}$, específicamente, en un incremento en defectos de la cabeza de espermatozoides en la especie humana (Morte et al., 2008); sin embargo, en este estudio no se encontró correlación significativa entre estos dos parámetros (Cuadro 4). 
La oxidación proteica tuvo efectos significativos sobre MT, MP, VSL, VCL, VAP, ALH y VE (Cuadro 2; $<<0.05$ ); y un coeficiente de correlación positivo para la morfología anormal (Cuadro 4). Son escasos los reportes que evalúan la oxidación de proteínas en el plasma seminal equino. Morte $e t$ al. (2008) evidenciaron una correlación positiva entre los niveles de oxidación de lípidos y proteínas en los espermatozoides y en el plasma seminal, con la presencia de defectos severos en la función de los espermatozoides de equinos subfértiles, indicando que la oxidación de lípidos y proteínas podría ser útil para la identificación de machos con problemas de fertilidad. Para la especie humana, se ha evidenciado un incremento significativo en el nivel de grupos carbonilos, generados como consecuencia de la oxidación proteica, en el semen de pacientes oligoastenoteratozoospérmicos en comparación con pacientes fértiles $(\mathrm{p}<0.001)$; asimismo, altos niveles de grupos carbonilos se han correlacionado positivamente con anormalidades del axonema del espermatozoide, con una consecuente disminución de su movilidad progresiva (Moustafa et al., 2009).

\section{Conclusiones}

Se concluye que los niveles de vitamina $\mathrm{C}$, proteínas totales y oxidación del plasma seminal están asociados con la criotolerancia del semen equino.

\section{Literatura Citada}

1. Aitken RJ. 2006. Sperm function tests and fertility. Int J Androl 9: 69-75. doi: 10.1111/j.1365-2605.2005.00630.x

2. Alghamdi AS, Troedsson MH, Xиe JL, Crabo BG 2002. Effect of seminal plasma concentration and various extenders on post-thaw motility and glass woolSephadex filtration of cryopreserved stallion semen. Am J Vet Res 63: 880885. doi: 10.2460/ajvr.2002.63.880
3. Aurich JE, Kühne A, Hoppen H, Aurich C. 1996. Seminal plasma affects membrane integrity and motility of equine spermatozoa after cryopreservation. Theriogenology 46: 791-797. doi: 10.1016/S0093-691X(96)00237-3

4. Ball BA, Gravance CG, Medina V, Baumber J, Liu IKM. 2000. Catalase activity in equine semen. Am J Vet Res 61: 1026-1030. doi: 10.2460/ ajvr.2000.61.1026

5. Ball BA. 2008. Oxidative stress, osmotic stress and apoptosis: impacts on sperm function and preservation in the horse. Anim Reprod Sci 107: 257-267. doi: 10.1016/j.anireprosci.2008.04.014

6. Barth AD, Oko RJ. 1989. Abnormal morphology of bovine spermatozoa. USA: Iowa University Press. 285 p.

7. Baumber J, BallBA, Gravance CG, Medina V, Davies-Morel MC. 2000. The effect of reactive oxygen species on equine sperm motility, viability, acrosomal integrity, mitochondrial membrane potential and membrane lipid peroxidation. J Androl 21: 895-902.

8. Bradford MM. 1976. A rapid and sensitive method for the quantitation of microgram quantities of protein utilizing the principle of protein-dye binding. Anal Biochem 72: 248-254. doi: 10.1016/00032697(76)90527-3

9. Brito L, Greene L, Kelleman A, Knobbe M, Turner R. 2011. Effect of method and clinician on stallion sperm morphology evaluation. Theriogenology 76: 745-750. doi: 10.1016/j.theriogenology.2011.04.007

10. Brum A, Sabeur K, Ball, B. 2008. Apoptotic-like changes in equine spermatozoa separated by density-gradient centrifugation or after cryopreservation. Theriogenology 69: 1041-1055. doi: 10.1016/j.theriogeno-logy.2008.01.014

11. Bustamante Filho IC. 2006. Estresse oxidativo na criopreservação do sêmen equino. MSc Thesis. Porto Alegre: Faculdade de Agronomia, Universidade Federal do Rio Grande do Sul. 90 p. 
12. Bustamante Filho IC, Pederzolli CD, Sgaravatti AM, Gregory RM, Dutra Filho CS, Jobim MIM, Mattos RC. 2009. Skim milk-egg yolk based semen extender compensates for non-enzymatic antioxidant activity loss during equine semen cryopreservation. Anim Reprod 6: 392-399.

13. Fagan JM, Sleczka BG, Sohar I. 1999. Quantitation of oxidative damage to tissue proteins. Int $\mathrm{J}$ Biochem Cell B 31: 751-757. doi: 10.1016/S13572725(99)00034-5

14. Fagundes B. 2003. Análise do congelamento e descongelamento de sêmen eqüino utilizando proteínas do plasma seminal em diferentes concentrações. MSc Thesis: Brazil: Universidade Estadual do Norte Fluminense. 47 p.

15. Gamboa $S$, Rodrigues $A S$, Henriques L, Batista C, Ramalho-Santos J. 2010. Seasonal functional relevance of sperm characteristic in equine spermatozoa. Theriogenology 73: 950-958. doi: 10.1016/j.theriogenology.2009.11.023

16. García BM, Fernández LG, Ferrusola CO, Salazar-Sandoval C, Rodríguez AM, Martinez HR, et al. 2011. Membrane lipids of the stallion spermatozoon in relation to sperm quality and susceptibility to lipid peroxidation. Reprod Domest Anim 46: 141-148. doi: 10.1111/j.1439-0531.2010.01609.x

17. Giesecke K, Sieme H, Distl O. 2010. Infertility and candidate gene markers for fertility in stallions: a review. Vet $\mathrm{J} 185$ : 265-271. doi: 10.1016/j.tvj1.2009.07.024

18. Graham J, Mocé E. 2005. Fertility evaluation of frozen/thawed semen. Theriogenology 64: 492-504. doi: 10.1016/j.theriogenology.2005.05.006

19. Guasti PN, Monteiro GA, Papa FO. 2012. Componentes do plasma seminal e sua influência sobre a criopreservação e fertilidade de espermatozoides equinos. Vet Zootec 19: 169-180.

20. Guasti PN, Souza FF, Scott C, Hartwig FP, Papa, MP, Dos Santos GAM, et al. 2014. Protein content of equine seminal plasma and sperm plas- ma membrane in subfertile stallions. J Equine Vet Sci 34: 84-85. doi: 10.1016/ j.jevs.2013.10.057

21. Heckenbichler S, Deichsel K, Peters $P$, Aurich C. 2011. Quality and fertility of cooled-shipped stallion semen at the time of insemination. Theriogenology 75 : 849-856. doi: 10.1016/j.theriogenology.2010.10.027

22. Hidalgo M, Rodriguez I, Dorado J, Sanz J, Soler C. 2005. Effect of sample size and staining methods on stallion sperm morphometry by the Sperm Class Analyze. Vet Med-Czech 50: 24-32.

23. Kankofer M, Kolm G, Aurich J, Aurich C. 2005. Activity of glutathione peroxidase, superoxide dismutase and catalase and lipid peroxidation intensity in stallion semen during storage at $5{ }^{\circ} \mathrm{C}$. Theriogenology 63: 1354-1365. doi: 10.1016/j.theriogenology.2004.07.005

24. Kareskoski M, Katila T. 2008. Components of stallion seminal plasma and the effects of seminal plasma on sperm longevity. Anim Reprod Sci 107: 249-256. doi: 10.1016/j.anire-prosci. 2008.04.013

25. Katila T, Anderson M, Reilas T, Koskinen E. 2002. Post-thaw motility and viability of fractionated and frozen stallion ejaculates. Theriogenology 58: 241-244. doi: 10.1016/S0093691X(02)00783-5

26. Kirschvink N, De Moffarts B, Lekeux P. 2008. The oxidant/antioxidant equilibrium in horses. Vet J 177: 178-191. doi: 10.1016/j.tvj1.2007.07.033

27. Madeiros A, Gomes G, Carmo M, Papa F, Alvarenga M. 2002. Cryopreservation of stallion sperm using different amides. Theriogenology 58:273-276. doi: 10.1016/S0093-691X(02) 00898-1

28. Michael AJ, Alexopoulos C, Pontiki EA, Hadjipavlou-Litina DJ, Saratsis PH, Ververidis HN, Boscos CM. 2008. Quality and reactive oxygen species of extended canine semen after vitamin $\mathrm{C}$ supplementation. Theriogenology 70 : 827-835. doi: 10.1016/j.theriogenology. 2008.05.043 
29. Moein MR, Dehghani VO, Tabibnejad $N$, Vahidi S. 2007. Reactive oxygen species (ROS) level in seminal plasma of infertile men and healthy donors. Iran J Reprod Med 5: 51-55.

30. Moore AI, Squires EL, Graham JK. 2005. Effect of seminal plasma on the cryopreservation of equine spermatozoa. Theriogenology 63: 2372-2381.

31. Morrell JM, Georgakas A, Lundeheim N, Nash D, Davies MCG, Johannisson A. 2014. Effect of heterologous and homologous seminal plasma onstallion sperm quality. Theriogenology 82: 176-183. doi: 10.1016/j.theriogenology.2014.03.020

32. Morte MI, Rodrigues AM, Soares D, Rodrigues AS, Gamboa $S$, RamalhoSantos J. 2008. The quantification of lipid and protein oxidation in stallion spermatozoa and seminal plasma: seasonal distinctions and correlations with DNA strand breaks, classical seminal parameters and stallion fertility. Anim Reprod Sci 106: 36-47. doi: 10.1016/ j.anireprosci.2007.03.020

33. Moustafa AA, El-Taieb Herwig R, Nada EA, Greilberger J, Marberger M. 2009. Oxidative stress and epididymal sperm transport, motility and morphological defects. Eur J Obstet Gynecol Reprod Biol 144: 199-203. doi: 10.1016/j.ejogrb.2009.02.018

34. Neild D, Chaves G, Flores M, Mora N, Beconi M, Agüero A. 1999. Hypoosmotic test in equine spermatozoa. Theriogenology 51: 721-727. doi: 10.1016/S0093-691X(99)00021-7

35. Nichi M, Bols PE, Zuge RM, Barnabe VH, Goovaerts IG, Barnabe RC, Cortada CN. 2006. Seasonal variation in semen quality in Bos indicus and Bos taurus bulls raised under tropical conditions. Theriogenology 66: 822-828. doi: 10.1016/j.theriogenology. 2006.01.056

36. Nouri M, Ghasemzadeh A, Farzadi L, Shahnazi V, Ghaffari M. 2008. Vitamins $C, E$ and lipid peroxidation levels in sperm and seminal plasma of asthenoteratozoospermic and normo zoospermic men. Iran J Reprod Med 6: 1-5.

37. Novakova L, Solich P, Solichova, D. 2008. HPLC methods for simultaneous determination of ascorbic and dehydroascorbic acids. Trends Anal Chem 27: 942-958. doi: 10.1016/ j.trac.2008.08.006

38. Ortega FC, González FL, Morrell JM, Salazar SC, Macías GB, RodríguezMartinez H, et al. 2009. Lipid peroxidation, assessed with BODIPY$\mathrm{C} 11$, increases after cryopreservation of stallion spermatozoa, is stallion dependent and is related to apoptotic-like changes. Reproduction 138: 55- 63. doi: 10.1530/ REP-08-0484

39. Parks J, Graham J. 1992. Effects of cryopreservation procedures on sperm membranes. Theriogenology 38: 209-222. doi: 10.1016/0093-691X(92)90231-F

40. Pesch S, Bergmann M, Bostedt H. 2006. Determination of some enzymes and macro- and microelements in stallion seminal plasma and their correlations to semen quality. Theriogenology 66:307-313.

41. Pessanha MA, Straggiotti JF, Fagundes B, Costa JR, Valente de Souza G, Shimoya A. 2008. Efeito de proteínas do plasma seminal eqüino com massa superior a $10 \mathrm{kDa}$ concentradas 10 vezes sobre a congelabilidade do sêmen. Rev Bras Zootec 37: 2115-2119. doi: 10.1590/S1516-35982008001200006

42. Pizarro LE, Restrepo BG, Echeverry ZJ, Rojano B. 2013. Efecto del plasma seminal sobre el estado redox del semen equino criopreservado. Rev MVZ Córdoba 18: 3672-3680.

43. Rigby SL, Brinsko SP, Cochran M, Blanchard TL, Love CC, Varner DD. 2001. Advances in cooled semen technologies: seminal plasma and semen extender. Theriogenology 68: 171-180. doi: 10.1016/S0378-4320(01)00154-3

44. Serafini-Cessi F, Cessi C. 1968. Thiobarbituric acid test as an index of phospholipid peroxidation. Sperimentale 118: 371-378. 
45. Vasconcelos J, Chaveiro A, Góis A, Moreira da Silva F. 2013. Effects of a-tocopherol and ascorbic acid on equine semen quality after cryopreservation. $\mathrm{J}$ Equine Vet Sci 33: 787-793. doi: 10.1016/ j.jevs.2012.12.012

46. Waheed M, El-Bahr SM, Al-haider AK. 2013. Influence of seminal plasma antioxidants and osteopontin on fertility of the Arabian horse. J Equine Vet Sci 33: 705-709. doi: 10.1016/j.jevs. 2012. 11.006

47. Warren L. 2009. Feeding the stallion. Canada: Alberta Agriculture and Rural Development. 4 p.

48. Watson PF. 2000. The causes of reduced fertility with cryopreserved semen. Anim Reprod Sci 60-61: 481-492. doi: 10.1016/S0378-4320(00)00099-3 